\title{
Green House Control System: A Research
}

\author{
Alka Singh, Ashutosh Kumar Singh
}

\begin{abstract}
Agriculture now is shifting in light of society's requirements. Where promising to provide as an instance, drinking water retention implanted area, which ensure superb services and products are very popular. Green houses have become for accomplishing those targets an answer; afterward back, problems that were tough can be prompted by a greenhouse for a technique for the farming. The increased section of these are discovered within the greenhouse setting states by which restraining the temperatures, and relative humidity $(\mathrm{RH})$ would be the essential targets of technology. Accomplishing climate states that were proper to ensure that usage diminishing are the target of search for a significant long time. Programs in manage hypotheses are connected to manage the topics previously. The idea with the paper is to introduce an overview of control strategies that are diverse implemented in farming to manage rainwater setting requirements, revealing detriments and conditions of get a grip on levels that are to indicate a design approach according to outcome gained by assessments.
\end{abstract}

\section{INTRODUCTION}

Rainwater is an enclosed room that produces a separate environment compared to this detected out as a result of confinement of this atmosphere also into the absorption of short- wave solar power by means of vinyl or plastic addresses (El Ghoumariet al., 2005). Rainwater might be understood to be a coated construction that delivers crops with all a brilliantly regulated atmosphere for alteration of climate-growth states, to lower the amount being spent on the creation and boost harvest yields (Badgery- Parker, 1999). That creates a new environment within the greenhouse that is greater called micro-climate. The rainwater micro climate might be manipulated by handle activities, for example heating, and venting, $\mathrm{CO} 2$ enrichment to list a couple; to present appropriate ecological requirements (Bennis et al., 2008). These alterations suggest the utilization of power from the manufacturing procedure. What's more, it needs a handle system that reduces the electricity consumption whilst keeping their condition factors as long as you can to this best harvest physical benchmark (Coelho et al., 2005). Horticulture in suburban states can be rising in its own societal and economical worth and is just really an interest. Improvements through the nineties are examined by written and Bailey (2002). Greenhouse structure's masking substance may fluctuate from simple sheets of transmission medium for various frequencies, that moisturizes equally vegetation zone along with its own surroundings and also drives electricity within the greenhouse. The expense of rising in a greenhouse is commonly more compared to climbing from the discipline; hence automation and

Revised Version Manuscript Received on 10 September, 2019.

Alka Singh, Information Technology, Noida Institute of Engineering and Technology, Noida, U.P. India. (Email: research.paper@niet.co.in)

Ashutosh Kumar Singh, Electronics and Communication Engineering, Noida Institute of Engineering and Technology, Noida, U.P. India. (Email: research.paper@niet.co.in) monitoring manage of applicable ecological parameters like air temperature, relative humidity $(\mathrm{RH})$, moderate amount and $\mathrm{CO} 2$ focus are needed to attain high return at a very reduced expense and also to continue to hold the surroundings aggressive (Bailey, 2002). In addition, it's essential that water and nutrients to be sent. Identification and modeling of many distinct parameters in greenhouse manufacturing have already been themes of countless research workers, for example Bot (1983), Hashimoto et al., (1993), Zhang al., (1997), Bekkaoui, (1998) and Bakker, respectively (2006). Atmosphere plant manufacturing methods give the option to present increased predictability to huge quantities of plants.

The world people have shifted food process continues to be all on. At a manner that is similar, ventilation and cooling ought to Production scenario within the a long time. Growing Nations, largely in subtropical and tropical area, promote and therefore are Predicted to Keep on supplying as Large as approximately 90 percent of Earth population growth (Soni along with Salokhe, 2004). Land place in 91 growing states, that isn't being used for harvest production is 2.4 times more compared to the place in usage (FAO, 2002). Because the territory that was readily available can't grow, greenhouse manufacturing was working to produce more effective usage of distance on. Several style processes for building of neighborhood shelters in tropical surroundings can be found from both Zabeltitz and Baldwin (1999). Efforts are forced to come up with computerized rainwater environment control approaches. Specifically, intriguing and relevant best control procedures are suggested (Ioslovich et al., 2009).

\section{STEPS OF AUTOMATION IN GREENHOUSE}

Even though idea of control and automation system at the rainwater would be just like its software, you can find borders that different those 2 areas of their controller. Individual ecological and biological detectors whereas other management and instrumentation supplies with the capacity of employed in an exact humid setting but not moving from calibration, along using a algorithm to adapt itself into diverse lively situations depending on special evolving phases, microclimatic variables and harvest's conditions as properly as considering geographic and pure elements, are exactly what create the rainwater controller not the same as industrial automation and command[1].Generally, automation at a tropical climates surroundings calls for automation of local weather controller (get a handle on atmosphere temperature, air flow and atmosphere market, $\mathrm{RH}$ controller and direction ), lighting amount controller and color drape direction, $\mathrm{co}_{2}\left(\mathrm{CO}_{2}\right)$ controller, irrigation, and

Published By:

Blue Eyes Intelligence Engineering 
compound treatment along with nutritional distribution administration. The expression environment means the crops. Greenhouse Automation is exactly about a highly authentic, effective and contemporary intensive agriculture, that judicially utilize accessible sources, recycles the data also maintains productivity, better yields whilst remaining environmentally. Greenhouse Automation is correlated with direction during the marketing procedure [2]. App of the incorporated system for the building's energy efficient automation from the greenhouse was shared with Marinakis et al., (2013). Even a classical and easy management program for climates includes detectors, each indoors and out, which track all significant environmental factors which impact crop development. These detector data responses to a control. The parameters below controller is going to be changed as a result of actuators these like coolers, buffs technique, lights process and also $\mathrm{CO}_{2}$ sprayers along with pre-defined instructions. It Ought to be conducted in an manner that is incorporated such that $\mathrm{CO}_{2}$ Isn't injected While the venting Be dispersed without even throwing away energy to restrain humidity and temperature. Even though utilizing at least two collections of separate detectors can also ensure the truth of technique observation and controller, however, the truth is, vegetation may perhaps not mandatory practical encounter a tightly managed natural surroundings (Bailey, 2002).Even though numerous environmental command versions are at present available that correlate input signal and input of the specific greenhouse for both simulation or simulation search intentions (Boulard and Baille, 1995; Rodríguez et al., 2002; Segineret al., 1994; Sigrimis and Rerras, 1996), however also a greenhouse command algorithm could possibly become a composite of various control techniques and strategies, (for example feed forwards, classical suggestions using proportional-integral-derivative, self-tuning and elastic, multivariable, measure or incorporated, etc.. ) to take care of some complicated and dynamic conditions by which maybe perhaps not even a overall version can be found. The reduction of the growth of this end rate or this temperatures beyond the greenhouse will cause the decline of temperature," resulting in a big change while inside greenhouse climate's RH [3]. Inside this circumstance, the feed-forward control (or anticipatory controls), is utilized in the event the controller answer is foreseeable for any particular pair of requirements. Energy-balance specimens are often used to compute the consequence which this system's condition gets got the parameter. The actuators are triggered before your deviation. Greenhouse units really are everywhere, hence, important until impacts are felt within the glasshouse that restrain signs might be routed. Method can decrease errors until they've enough the time to impact the body until changes from place point does occur detectors quantify the launching of ports and also correct temperature controller since the clogs are quantified. Rodríguez et al., (2001) intended a feedforward control for greenhouse weather controller predicated on physiological versions. A higher level of accuracy may be accomplished Wherever feedback modification is employed in combination with feed controller. At an feedback controller, your choice depends upon differences among your dimensions and also the inputs that were desirable; hence a variation is anticipated until almost any reply can be provided by the control that occurs.

The output signal will likely probably be oscillating and slow above and under [4]. At percentage, the answer has been delivered At an controller to just how a long way the state that was quantified is all. Cool drinking water can be started between complete and full off for the malfunction involving the crop and also comfortable zone temperature. Control profits that are smaller are produced by errors. In percentage, the answer has been sent Within an controller to this period that a factor has deviated from the own price. In case the remarks mistake is smaller however, has been making for many moment, the advantage that is key induces raising the pace of answer. Essential and proportional rainwater construction and masking substances, Controller to extend a way of returning a state into the place - purpose management a reaction to the level of deviation along with the sum of time which the deviation has happened. In case the speed of shift from the quantified factor is accelerated, private manage may be utilized in combination with proportional and integration calculation to shorten time that it requires to adjust a controller variation, but this activity will be infrequently necessary for rainwater equipment expected to additional procedure metering [5]. Measure controller can be utilized to disperse controller answers from several unobtrusive measures. At a greenhouse as an instance, it may be desired to give a lot greater than 1 venting platform by implementing venting followers that, should the temperature climbs a level previously mentioned plant relaxation level, state $3^{\circ} \mathrm{C}$, the lover is started by then the control. In the event the temperatures is over $6^{\circ} \mathrm{C}$ yet still another lover begins. The answer behaves in-full length performance, just about every buff will be on or off, however in a incorporated controller, answer can normally be proportioned within the full interval between complete / away, supplying an even more precise controller response together using interdependent connections along with numerous impacts of the greenhouse gear considering consideration. The parameters to adjust by on their own for optimizing performance and reducing mistakes inside the technique that is regulated [6]. This way is utilised in conjunction with intelligence including Genetic Algorithm and Fuzzy Logic. An use of this self- functioning procedure for this celestial procedure on greenhouse procedure might be researched from the job of Aoud et al.," (2007). Within a flexible control, self-tuning controllers' features have carried a stage farther. In the event the parameters of the managed technique are unclear or fluctuate, then the management legislation in a flexible controller permits the controller parameters to be adapted regularly into the shifting requirements (Aström and Wittenmark, 1995). No accounts should be removed from the connections among active factors, In case just a single output factor is usually always to be commanded with just a variable that is controlled. A rainwater management procedure, but usually do not adapt to this type of small configuration, as an instance, the consequence in fever if managing humidity asks a method with a lot greater than 1 control loop, also called as multivariable controller programs, that employs a version of this controller surroundings for offering advice regarding the 
connections. Arvanitis et al.,(2000) intended a Multi-rate flexible temperature controller of green houses.

\section{IDEAL CONDITIONS \& RESULTS}

As Stated by the graph, RHare and temperatures Inter-related. Which usually means that the temperature of some volume of atmosphere increases this air's power and affects its RH. Atmosphere will diminish RH and limiting condensation will come about from heating down it. Even the Psychrometric graph proves that $\mathrm{RH}$ wills drop. $\mathrm{RH}$ and Infection have collections predicated upon growth phase of the plant farmers harvest, so also weather illness and also if it's day or night. A decision support process to its creation of veggies that are high grade can be found from the Ohio agricultural research and development center. Namely, scientific processes usefulness principle, hazard appraisal, and selection aid theory have now been found to encourage real harvest growing tips which could be drawn up from worth. The app can be obtained in 2 manners, being a primer that is graphic so that within an interactive decision aid platform [7]. This harvest inside this program's definition is really actually just a high return. But on the flip side, a plant can make reference to substantial return or low carb harvest. The best worth for the tomato in weather and mild state, also for a particular development period. The amount for humidity is based upon the sort of crop to be increased. Even the American culture of heating and air-conditioning Engineers (ASHRAE) believes RH in between 40 percent and 60 percent nutritious and comfy in relaxation - managed surroundings (ANSI/ASHRAE, 2010). An industrial greenhouse provider (Autogrow programs, Ltd.; Entrance:// www. Autogrow.com / / general-info/humidity-and-vpd. Accessed on: May 2014) defines RH vary between 60 percent and 80 percent to become more comfortable to greenhouse plants humidity is required by vegetation vulnerable to temperature. This really is regarding the plant transpiration from the plant proceeds to re grow longer escalating $\mathrm{RH}$ of those air will lessen transpiration, deferring the wilting level. In Contributes to heat leaking in the pay, inducing aside from seeming nutrient deficiencies because of weaksap movements from the 22, spores life. An excessive amount of transpiring can be additionally stopped by RH and shed tumor. Vapor Pressure Deficit or VPD may be your gap (shortage) involving your quantity of dampness from the atmosphere and the amount of moisture the air can endure if it's saturated. Once that the atmosphere gets soaked, water tends to float outside to produce pictures, dew or clouds of plain water. It's the previous example which produces VPD mandatory for greenhouse regulation[8]. It gets a lot more vulnerable to rust When a picture of water creates in the plant foliage. Since the VPD advances, About the flip side the plant should draw water. The plant perish and can dry. Because of this the perfect choice for VPD at a rainwater would be out of $0.45 \mathrm{kPa}$ into $1.25 \mathrm{kPa}$, preferably sitting down around $0.85 \mathrm{kPa}$. Like a rule of thumb, many plants develop well in VPDs of involving 0.8 to $0.95 \mathrm{kPa}$. VPD may function from the atmosphere as an indicator of humidity shortage. This is of VPD relies upon fever and RH, which are with the capacity of improved symbolizing the way the plant believes (Collier et al.," 2000). Since Compounds infect and grow vegetation VPD is utilized to quantify the environment will be always to glow. One other sign of VPD will be also to spot whether it's very likely to occur and also always to appraise heating of the greenhouse harvest. As stated by the mention, fungal infections and nutrient deficiency signs and indicators arise beneath VPD worthiness of $0.43 \mathrm{kPa}$ and disorder illness could be damaging below VPD worthiness of $0.2 \mathrm{kPa}$. It's thus suggested the VPD of rainwater atmosphere ought to really be held over $0.20 \mathrm{kPa}$. The perfect array of VPD for all rainwater plants is amongst 0.8 to $0.95 \mathrm{kPa}$ (Argus control-systems, 2009). Adding humidity into the atmosphere is obligatory for worth significantly more than $1.25 \mathrm{kPaheat}$ and dehumidification needs to be implemented for VPDs beneath $0.45 \mathrm{kPa}[9]$.

\section{CONCLUSION}

Environment controller is now one of the goals of technology from precision agriculture. Humidity and Stress are all. Current analyses have demonstrated that it's perhaps maybe not sufficient to correct humidity and temperature evaluations for minimum and maximum set points which can be cheap for older plant requirements. Best control methods rely primarily on AI calculations and flexible control concept that propose an alternate approach to fix the local weather controller problem if calculating version is often intricate or unknown. One other fact, that's constrained the evolution of the climate management system that is complex level has been that the limits. Nevertheless, the brand-new technology like digital signal processors, microprocessors and field programmable gate arrays are permitting ongoing the execution of controller procedures that are complex. As stated by a few writers, the use of controls of after factor set-points have never proven to become an ideal option. As a result with the fresh trends have been emerging in marine climate controller in line with collecting info regarding morphological and bodily procedures inside the plant stomatal conductance and photosynthesis. These controller concepts that are brand new to report it isn't necessary to own temperature and humidity collection points. Rather than that thresholds have been suggested to store power consumption that is absorbed while the controller attempts to stick to precisely the stage in a way. Photocontrol could be your principle that indicates this plant reactions within an input sensor's use to set up the point from the local weather control. Additionally, it have never been shown to become quite a way as it's critical to collect info to establish that the visibility with this. Different types of controls have surfaced revealing pitfalls and benefits much far superior functionality for a number of activities along with other faculties, of these. Researchers will want to test various control concepts to decide on what type would be your very proper to their endeavors, in line with their own essentials of greenhouse environment control methods. This paper offered a debate of their degrees, this controller factor and interaction. The automation degrees and also restrain that is different strategies that are in the exploration and also experimentation periods or have already now been commercialized to-use at green houses were discussed. It might be reasoned that

Published By:

Blue Eyes Intelligence Engineering 
knowledge to plant structure and ecological fluctuations in tropical oceans collectively with assessing both the handle parameters along with their connections together using the environment, technology modeling, simulation theories and get a handle on hardware/ applications substances, encourage a much better and more successful design of their automation management applications / hardware method which may satisfactorily handle and track environmental parameters such as data investigation and assessing functionality.

\section{REFERENCES}

1. S. Wang, S. Wang, and J. Liu, "Life-cycle green-house gas emissions of onshore and offshore wind turbines," J. Clean. Prod., vol. 210, pp. 804-810, Feb. 2019.

2. Y. Noh, "Does converting abandoned railways to greenways impact neighboring housing prices?," Landsc. Urban Plan., vol. 183, pp. 157-166, Mar. 2019.

3. V. Tavares, N. Lacerda, and F. Freire, "Embodied energy and greenhouse gas emissions analysis of a prefabricated modular house: The "Moby\{"\} case study," J. Clean. Prod., vol. 212, pp. 1044-1053, Mar. 2019.

4. Y. Hwang, B. Chang, T. Kim, and S. Kim, "Ameliorative effects of green tea extract from tannase digests on house dust mite antigen-induced atopic dermatitis-like lesions in NC/Nga mice," Arch. Dermatol. Res., vol. 311, no. 2, pp. 109-120, Mar. 2019.

5. J. L. S. Pereira, S. Ferreira, V. Pinheiro, and H. Trindade, "Ammonia and greenhouse gas emissions following the application of clinoptilolite on the litter of a breeding hen house," Environ. Sci. Pollut. Res., vol. 26, no. 8, pp. 8352-8357, Mar. 2019.

6. J. Lee and M. Shepley, "THE GREEN STANDARD FOR ENERGY AND ENVIRONMENTAL DESIGN (G-SEED) FOR MULTI-FAMILY HOUSING RATING SYSTEM IN KOREA: A REVIEW OF EVALUATING PRACTICES AND SUGGESTIONS FOR IMPROVEMENT," J. GREEN Build., vol. 14, no. 2, pp. 155-175, 2019.

7. Y. Ye, H. Xie, J. Fang, H. Jiang, and D. Wang, "Daily Accessed Street Greenery and Housing Price: Measuring Economic Performance of Human-Scale Streetscapes via New Urban Data," SUSTAINABILITY, vol. 11, no. 6, Mar. 2019.

8. A. C. Serrenho, M. Drewniok, C. Dunant, and J. M. Allwood, "Testing the greenhouse gas emissions reduction potential of alternative strategies for the english housing stock," Resour. Conserv. Recycl., vol. 144, pp. 267-275, May 2019.

9. J. S. Jeon, "How housing market responds to greenbelt relaxation: Case of Seoul Metropolitan Area, South Korea," Land use policy, vol. 84, pp. 328-334, May 2019.

10. J. Marx, A. Schreiber, and P. Zapp, "Response to 'Life-cycle green-house gas emissions of onshore and offshore wind turbines'," J. Clean. Prod., vol. 219, pp. 3334, May 2019. 